ton. Thus any remedy that can with any certainty produce the effect of quickly opposing the dissolution of the blood, will, I believe, cure the patient. Mercury, when judiciously applied, has the effect of increasing and thickening the albuminous portion of the blood very quickly, producing a favorable re-action against the morbid state, particularly in cases of snake bites, when associated with some powerful tonic. It is the remedy I have found the most active and efficacious in all such cases I have treated in this country. The manner in which I use it has been described in a former communication to this Journal (page 120). In some cases opium has been added to the powders or pills; but $I$ think it is better to spare its use, and to avoid narcotization, as it is important to keep the patient excited and awake to facilitate re-action. Common drink-cold water, or any aromatic infusion, not in contradiction with the medicines. Nourishment, if indicated or possible-oatmeal gruel, arrowroot, or bread and tea, \&c.

I remember attending, some years ago, in Caracas, the cousin and overseer of Dr. Carlos Arvelo, an eminent and well-known physician, and at the doctor's own house, for the bite of a large and very poisonous snake called "mapanare." The bite was over the pedal artery; the case was a very severe one, with complete dissolution of the blood. Some bites of leeches which had been applied to him a month previous to the accident, for an affection of the stomach, opened suddenly, bleeding profusely; and I was obliged to cauterize every one of them with lunar caustic, introducing a small ball of lint in each hole with a probe, and placing some adhesive plaster over them. The patient recovered at last, under the very active and combined treatment above alluded to. The dose of calomel given every hour during three days, with very few exceptions, was eight grains, with ten of quinine, and some musk and camphor. Almost every grave symptom, indicated as general in such cases, presented itself; but I firmly persisted against advice in the stated treatment, and the patient was saved. Two years ago the patient passed through this city, with Dr. Arvelo's eldest son, now a physician also of Caracas, and called on me, saying he could not do less than renew his thanks for my past assistance. I examined the spot where he had been bitten by the snake. It still retained a livid appearance or darkred color of two inches square, although perfectly healed, the individual stating he felt now and then a dull pain in the foot.

I could refer to many other cases treated successfully on the same principles and with the same remedies; but it would occupy too much space to do so.

.Puerto Cabello, March 16th, 1851.

\title{
ON THE PRACTICAL USES OF COD-LIVER OLL.
}

[Communicated for the Boston Melical nud Burgical Journul.]

There is reason to believe that the uses of medicines are too much governed by the influence of popular excitement; and instead of thoroughly testing their intrinsic merits, as therapeutic agents, and retaining 
them, if valuable, for permanent resources, we too frequently throw them aside for other's that the tide of innovation has brought forward. Excepting a few well-known remedies, such as mercury, opium and antimony, which have been retained more on account of conservative prejudice than discriminating experience, this is a fact that cannot be denied ; and to this, is owing the history of thousands of medicinal agents, which one day had their votaries, and now only serve to sivell our materia medica into a ponderous and unwieldy tome. This has been the fate of medicines whose virtues were once demonstrated to be worthy the praise they then obtained. Instance the far-famed naphtha, which, but a few years since, was considered by the first practitioners almost a specific for phthisis pulmonalis, but is now nearly abandoned.

Shall this be the fate of cod-liver oil, now so extensively used as a remedy for scrofula and incipient pulmonary consumption? We have every reason to fear that it will be, because its great use was prematurely occasioned by popular excitement.

In no part of my professional experience, have I been more amused by the caprice of custom, than in introducing this article. Some three years since, being anxious to test its virtues in several cases, where all other reliable medicines had failed to benefit, 1 recommended a trial of it. At that time it was but little known; or, at any rate, it had not got into popular use: and although the flattering hope of patients under the peculiar exciting malady of consumption, is generally sufficient to induce a resort to anything new, the very name of fish oil was sufficient to excite a disgust against it, that I could not overcome in any other way than by reading to them several detailed reports of its wonderful cures, in Braithwaite's Retrospect, and other journals of the day.

But I commenced this article with the intention of giving the history of a few well-marked cases, showing the certain and happy results of the oil in my own practice.

Abijah Gleason, aged 50, of West Millbury ; occupation for several years has been that of grinding black lead. Temperament, equally of the nervous, sanguine and bilious, which has enabled him to sustain an unusual fortitude of mind. I was called to see him July $26,1848$. Much difficulty of respiration. He had been able to lie down but very little for three nights, owing to increased difficulty of respiration in the recumbent posture-a sharp catching pain in the anterior and lateral portion of right chest. Suddenly taken the night previous, raising, very rapidly, a large quantity of pure pus from the lungs, attended constantly with much soreness of the chest. Pulse 90, small. Appetite small and disturbed. Some cavernous cough, but not harassing; hectic, attended with colliquative night sweats, and alternate chills and febrile excitement. Percussion emits some dulness over inferior and lateral portion of right lung-dulness continuing from the lower third, so connected with the diaphragm and the situation of the upper portion of the right lobe of the liver, that it was difficult to tell how much of the dulness was occasioned by an enlargement or morbid position of the latter ; as tenderness as well as enlargement, together with morbid secretion, was discovered in the liver. Auscultation discovered evident pecto- 
riloquy; humid rhonchus and amphoric respiration at the summit of the right lung. A large cavity existed in this region-mucous or crepitant rales evidently indicating pulmonary fistula. Left lung resonant and healthy.

Patient says that he has had an abscess break as often as once a month for three years, and discharge to the amount of half a pint, or more, in a short time, and to continue a moderate expectoration from two or three days to a week in succession. If less frequently than once a month, the greater the shock and prostration produced upon the health and strength, and the more profuse the quantity discharged; and the more frequent these eruptions (and it is nothing unusual for them to return in much less than a month), the smaller the quantity expectorated, and the system more readily rallies from the effects. Much soreness, and a sense of distress or oppression, are always felt for two or three days before these discharges take place, so that he can always calculate on their appearance with considerable certainty. At times the prostration produced has been sufficient to confine him to the house for a week or two - at other times, especially if the weather is favorable, he may be able to take gentle exercise at once out of doors. Between these periods he is frequently able to walk a mile or more, without any great fatigue, and can ride several miles in an easy carriage without being uncomfortably affected. He had tried almost every system of treatment, from botanic or Thomsonian, down through every variety of nostrum that has been recommended or used for similar complaints, but without any permanent benefit, and probably with much injury.

At this time I considered the case incurable. The ulceration had been of so long standing, and the caverns in the lung were so large, it seemed but quackery to promise more than palliative relief. There being much tightness or stricture of the bronchi, and oppression, for the want of free expectoration to unload the lungs of the purulent accumulation; to fulfil this indication, and to stimulate the diseased surfaces to a healthy granulation, I administered, combined in suitable proportions, syrup lobelia, naphtha and morphia. 'To correct a feetid breath and loaded tongue, which I supposed proceeded from deranged secretions of the liver, in a measure, I gave occasionally a mild alterative-and likewise advised the liberal use of London porter. This treatment had a very beneficial effect for the time; he gradually recovered from the soreness and oppressed state of respiration, his strength rallied, so that he got out to his usual exercises, and attended to his business. But still, during the winter following, these paroxysms continued to occur with more and more severity, so that he became much reduced, and was confined for most part of the time to the house and to his room. At this time his life was despaired of both by himself and friends, and he made every arrangement in his business to meet soon the fatal issue. All treatment, thus far, seemed to be but of temporary benefit, while the ulceration appeared rapidly to be gaining ground. More with the idea of keeping up the hope of my patient, than the expectation of any sanative result, I advised a thorough trial of cod-liver oil. He commenced about the first of May, 1849, in doses of a tablespoonful three 
times daily, generally on an empty stomach, in porter. A month had not elapsed before a marked change had taken place both in his general health and the feeling of the lungs; the soreness gradually subsided, his cough disappeared, his respiration became easy and natural, and in a little time all expectoration of purulent matter ceased, together with the periodical eruptions. The hectic and night sweats abated, sleep became natural and refreshing; the appetite returned, with a healthy state of digestion; the muscles acquired fulness, with renewed tone and activity; and, in fact, the whole system, under the continued use of the oil, seemed to be regaining its wonted state of health. And now, after a period of nearly two years, there has not been an eruption or any appearance of a renewed abscess, and all purulent expectorations have long since subsided. He told me personally the last fall that he felt perfectly well; he had no trouble from his lungs, he felt in better health and stronger than he had done for years. He had worked steadily every day, and had, during the haying season, kept up with other good mowers, and done his day's work as easily as any laborer.

The beneficial results in this case must be attributed alone to the oil, as no other medicine was made use of from the time of its commencement. He has informed me, since discontinuing the oil, that he took some twelve bottles in all, holding a pint each. The question may justly arise in this case, whether the abscesses that formed so often and discharged in such large quantities, were the result of long-continued tubercular deposit or the immediate effects of pneumonia or bronchial inflammation? It is my opinion that they were the result of the latter, as he had always enjoyed good health up to the commencement of this attack, which was produced by a violent catarrh.

$$
\text { Worcester, April 1, 1851. [To he contlnued.] A. Stone, M.D. }
$$

[THE following essay, on a subject full of interest to the anatomist, was written and first published in 1832 . It is now re-printed, by request of the author, who wishes the reader to be apprised that he considers the subject very far from being exhausted-there still remaining an ample field for anatomical research. The "Supplement" was contained in the original essay.-ED.]

\section{AN ESSAY ON THE GANGLIONIC SYSTEM OF NERVES IN THE CAVITY OF THE CRANIUM, AND I'TS USE.}

BY WILJIAM INGALIS, M.D.

[Communicated for the Boston Medicul and Surgica] Journal.]

I. The energy of the brain, and the due performance of its functions, as it has been supposed, do not depend on its volume, the size of its convolutions, nor the temperaments.

II. As in other viscera, the perfect organism of the brain is undoubtedly a condition essential to the performance of its functions with exactitude and energy; for, a malconformation, or some defect in its minute 